\title{
Design and Application of Transmission Line Intelligent Monitoring System
}

\author{
Zengqiang Xing ${ }^{1 *}$, Wenpeng Cui ${ }^{1}$, Rui Liu ${ }^{1}$ and Zhe Zheng ${ }^{1}$ \\ ${ }^{1}$ Beijing Smartchip Microelectronics Technology Company Limited, Beijing 100080, China
}

\begin{abstract}
This paper presents a design method of intelligent monitoring system for transmission lines based on artificial intelligence technology. In this design method, a low-power artificial intelligence chip - LieYing A101 is used to design an intelligent recognition module to realize real-time target recognition on a terminal device. In order to solve the problem that the original image and the input image resolution of the intelligent recognition module do not match, this paper uses a sliding window and convolutional neural network design method, which solves the image resolution mismatch problem and improves the recognition accuracy. Finally, for the problem of excessive network model size, feature channel weight pruning and 8-bit quantization methods are used to compress the network model to less than $10 \mathrm{M}$, and the recognition accuracy is not sharply reduced. After the test set test and actual scene use, the external force destruction target recognition accuracy of the transmission line channel is high; this meets the application needs of customers.
\end{abstract}

\section{Introduction}

With the rapid development of power construction and the continuous expansion of the scale of the power grid, there is more and more work for power grid construction and equipment maintenance under complex terrain conditions. The transmission line as a power transmission link has the characteristics of wide dispersion, long distance, and is difficult to routing inspection. Therefore, the transmission line online monitoring system is an important part of the transmission link of the smart grid, and an important technical means to realize the transmission line status operation, maintenance management, and improve the level of operation management.

Taking the 2010 Hunan province power grid line fault as an example, there were 73 trips on $110 \mathrm{kV}$ and above lines, including 48 trips due to lightning strikes, and 14 trips caused by external force destruction, accounting for nearly $20 \%$ of the total trips. In addition to lightning strikes, the external force destruction is the most important cause of tripping of Hunan province power grid lines. The risks of external force destruction mainly include tower cranes, cranes, construction machinery (cement pumps, cement tankers, excavators, bulldozers, forklifts, road rollers, etc.), wildfire and smoke, line foreign bodies(kite, dust net, plastic film), etc. [1-2]

At present, there are two main methods for the inspection of transmission lines: One is the traditional manual inspection, mainly using auxiliary inspection equipment such as telescopes. The other way is to collect the images or videos of the transmission line by the transmission line monitoring equipment, and upload all the images or videos to the server through wireless communication. On the server side, the abnormal pictures are manually screened or automatically recognized by artificial intelligence algorithms.

The above two inspection methods have many shortcomings. The first method is the conventional manual inspection operation method, which is characterized by high work intensity, hard working conditions, and low efficiency. In case of emergency failure of power grid and abnormal climatic conditions, the inspection work cannot be carried out normally. The second method requires high network bandwidth for transmission line monitoring equipment, high network transmission pressure, huge data storage capacity, and expensive network transmission traffic.

This paper designs a transmission line intelligent monitoring device and system based on artificial intelligence technology, using low-power artificial intelligence chips to realize abnormal target recognition on the terminal side, which greatly reduces the network transmission pressure and reduces the load on the server. Improve the real-time nature of abnormal target recognition.

\section{System structure}

\subsection{Function introduction}


The transmission line intelligent monitoring device is generally installed on the angle iron of the high-voltage transmission line tower, and the installation angle of the monitoring device is adjusted according to the scope of the monitoring channel, the installation angle of the solar panel is adjusted according to the actual situation of the light [3].

The device periodically collects images of the monitored transmission line channel status, and the internal artificial intelligence module completes data inference to identify external force destruction in real time. If it is found that there is a risk of the external force destruction to the transmission line channel, the image and the recognition result are pushed to the system server, and the server finishes reporting the relevant information to the operation and maintenance staff, and at the same time starts early warning measures. The logic flow of the system is shown in Figure 1:

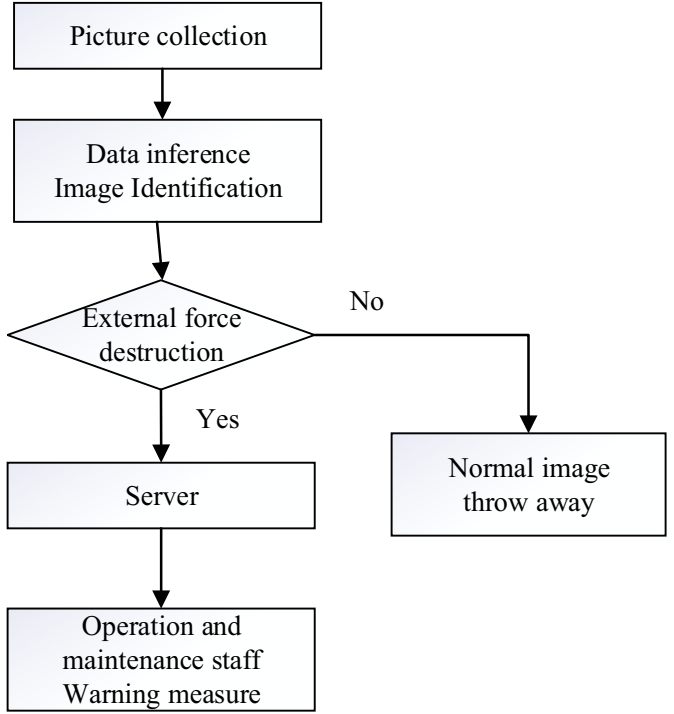

Figure 1. System logic flow chart

The system structure is shown in Figure 2:

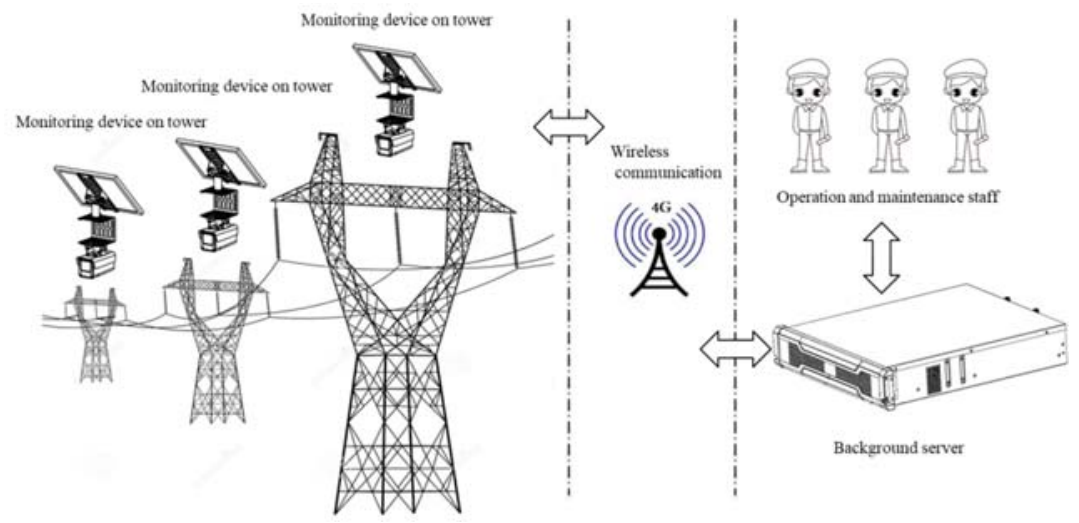

Figure 2. System components

\subsection{System composition}

The transmission line intelligent monitoring system is mainly composed of the monitoring device installed on the tower and the background server. The monitoring device on the tower mainly completes image acquisition, target recognition, and early warning linkage, including image sensor module, image processing module, intelligent identification module, control and storage module, wireless communication module, early warning linkage module, and power management module. The system is divided into functional modules as shown in Figure 3.

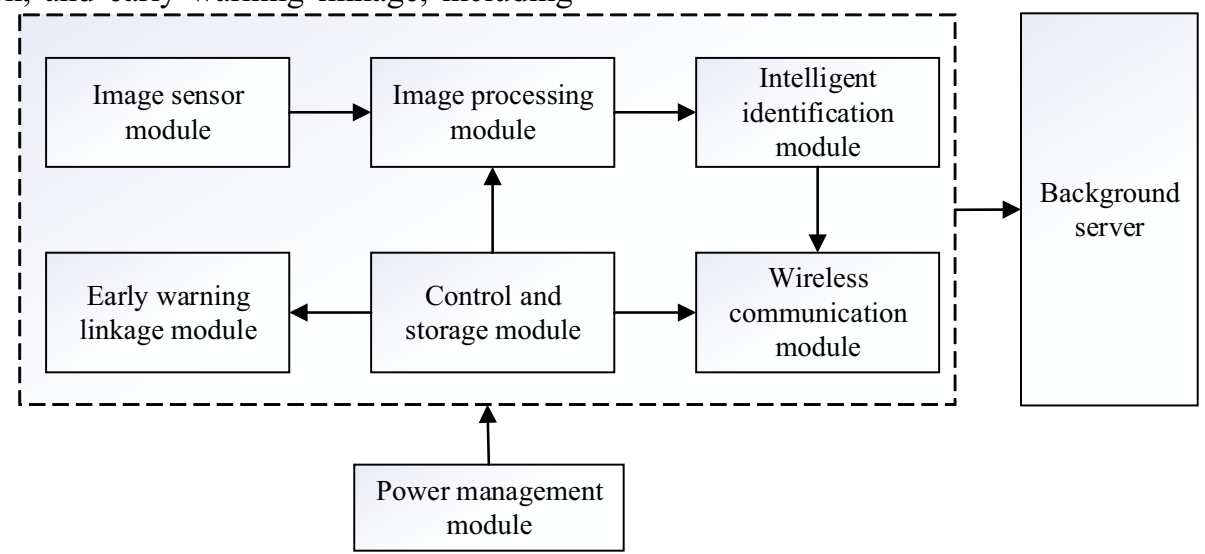

Figure 3. Function block diagram 
The image sensor module collects image data on transmission line channels in real time.

The image processing module receives the image data of the transmission line channel and completes the image coding and other functions. At the same time, according to the feedback results of the intelligent recognition module, complete frame synchronization and target category, coordinates, confidence and other information annotation.

The intelligent identification module, developed based on self-developed low-power artificial intelligence chip - LieYing A101, embedded with lightweight deep learning network model, can identify tower cranes, cranes, construction machinery (cement pump trucks, cement tank trucks, excavators) , Bulldozers, forklifts, rollers, etc.), wildfire smoke, line foreign bodies (kite, dust net, plastic film), etc.

The wireless communication module is used to upload abnormal image and video data to the server and at the same time receive the server configuration information and firmware/model upgrade data.

The control and storage module is used for the logical control of the monitoring device and the storage of data such as images, videos, logs, and firmware.

The early warning linkage module completes the sound and light linkage alarm.

The power management module adopts the power management scheme of solar panels, batteries and super capacitors, which greatly extends the life of the battery and ensures the stability of the overall system.

The background server mainly receives the abnormal data of the monitoring device and promptly pushes it to the relevant operation and maintenance staff. At the same time, all images and video data are classified and analysed.

\section{Key technologies}

\subsection{Intelligent identification module}

Based on the artificial intelligence chip - LieYing A101, the low-power artificial intelligence recognition module designed for end-side applications is suitable for intelligent analysis of videos and images, and can complete common image processing functions such as target detection, target recognition, and semantic segmentation. The peak power consumption of the module is not higher than $0.5 \mathrm{~W}$, the computing power is
1.4TOPS, and it supports USB2.0/USB3.0 protocol. Its functional block diagram is shown in Figure 4.

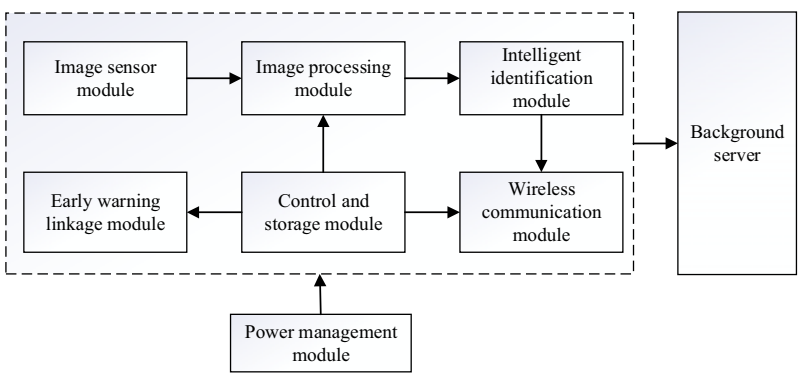

Figure 4. Functional block diagram

The operation of the module is mainly completed by two functions. The func_init() function completes the initialization of the algorithm model, including A101 chip initialization and internal data structure initialization. The func processImage () function calls the algorithm to process image data and returns the detection result, the resolution of the input image supports $224 * 224$, the returned result data includes category, confidence, and coordinate information.

\subsection{Sliding window and convolutional neural network}

At present, the images collected by the image sensor module are generally $200 \mathrm{~W}$ pixels, and even images with higher pixels of $400 \mathrm{~W}$ and $800 \mathrm{~W}$. The input image resolution of the intelligent recognition module is $224 * 224$. Forcing the original image to be cropped and compressed will cause image distortion and distortion, which seriously affects the recognition accuracy of the algorithm model. By designing a sliding window to traverse the original image, the partial images corresponding to each window are identified and detected, which effectively overcomes the problems caused by scale, position, deformation, etc. and improves the identification and detection effect.

Figure 5 shows the whole process of target detection on the original picture $(8 * 8)$ using sliding windows ( size $=6 * 6$, stride $=2$ ). The final output is a $2 * 2$ grid, and each grid corresponds to an output label vector, which gives the detection results (target category, confidence, coordinate information, etc.) of the window area image corresponding to the original picture. 


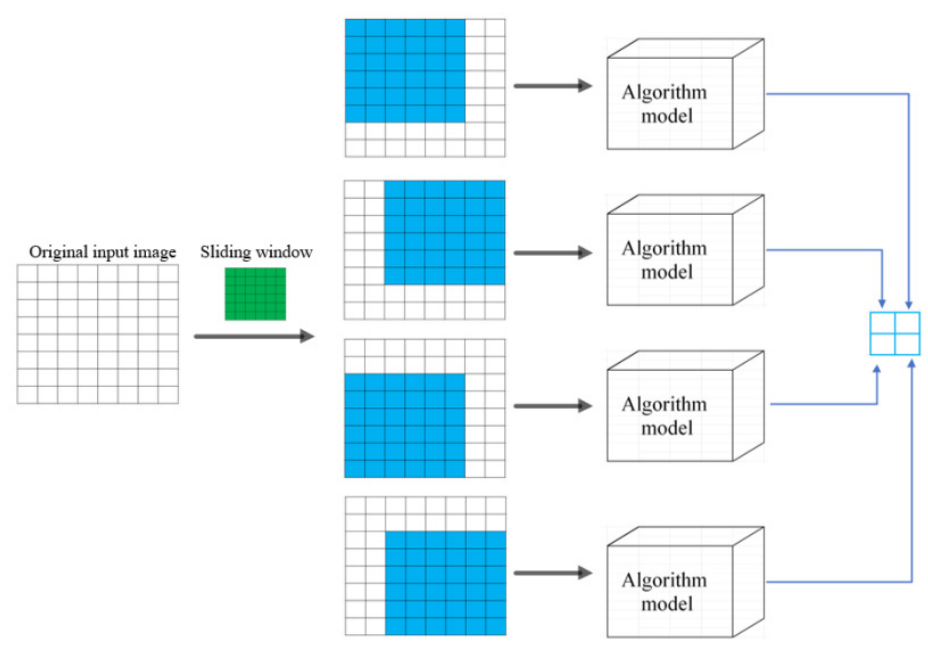

Figure 5. Sliding window

The algorithm model uses CNN (Convolutional Neural Network), which is the most widely used network model in deep neural networks. The characteristic of $\mathrm{CNN}$ is to obtain the eigenvalues of each layer through a convolution kernel that shares weights. A special structure makes $\mathrm{CNN}$ more suitable for the learning and expression of image features [4].

A typical CNN network structure is shown in Figure 6 , which is mainly composed of the convolutional layer, the pooling layer, and the fully connected layer. The convolutional layer is responsible for extracting local features in the image. The pooling layer is used to greatly reduce the magnitude of the parameters. The fully connected layer is to integrate image features through multiple convolutional layers and pooling layers for image classification.

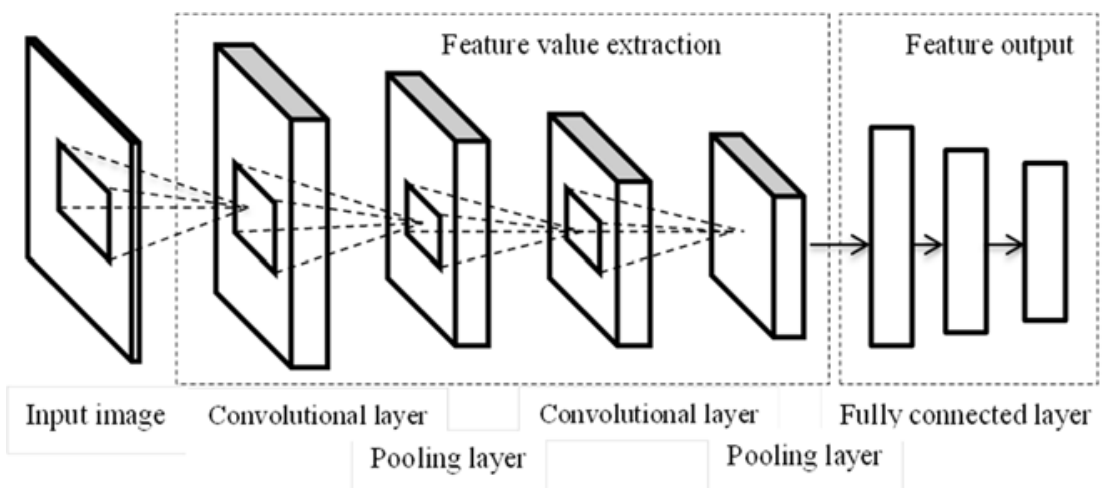

Figure 6. CNN network structure

The convolutional layer performs a convolution operation for the discrete $2 \mathrm{D}$ filter and the $2 \mathrm{D}$ image in image recognition, that is, the $2 \mathrm{D}$ filter slides to all positions on the 2D image, at each position the pixels of its field do inner product. In deep convolutional neural networks, low-level to complex image features can be extracted through convolution operations.

In the convolutional layer, it usually contains multiple learnable convolution kernels. The feature map outputted by the previous layer and the convolution kernel perform a convolution operation, that is, a dot product operation is performed between the input item and the convolution kernel, then the result is send to the activation function, you can get the output feature map. Each output feature map may be a combination of convolutional values of multiple input feature maps. The calculation of the output value $a_{j}^{l}$ of the $\mathrm{j}$-th unit of the convolution layer L is formula (1), where $M_{j}^{l}$ represents the set of selected input feature maps and $\mathrm{k}$ represents a learnable convolution kernel.

$$
a_{j}^{l}=f\left(b_{j}^{l}+\sum_{i \in M_{J}^{L}} a_{i}^{l-1} * k_{i j}^{l}\right)
$$

And dowm(.) is the pooling function, $b_{j}^{l}$ is the bias, $\beta_{j}^{l}$ is the multiplier residual, and $M^{l}$ is the size of the pooling frame used in the L-th layer is $M^{l} * M^{l}$

The pooling operation will keep the depth size unchanged. If the input unit' size of the pooling layer is not an integer multiple of two, the zero-padding method is generally adopted to become a multiple of two, and then pooled.

Fully-connected layer, which combines all local features into global features, is used to calculate the final score of each category. 


\subsection{Lightweight design of CNN model}

The convolutional neural network implements the target detection algorithm. Its network scale is usually above 100 megabytes, which requires a large amount of storage resources and computing resources, and has high operating power consumption, which cannot meet the application requirements of the side/end embedded terminals. Aiming at the target characteristics of the monitoring scene and the selected low-power artificial intelligence chip architecture, a special lightweight deep learning algorithm is designed, and the complexity of the model is further reduced through the trained pruning and quantization technology. On the premise of ensuring that the prediction accuracy is not degraded, the network model is compressed to below 10 megabytes.

Pruning scheme design. Theoretical research shows that the closer the weight is 0 , the smaller the corresponding neuron's influence on the prediction of the result. Eliminating redundant neurons will present effective neurons in a more compact form. A pruning algorithm based on feature channel weights is used to complete pruning of the model during the training process, and the purpose of reducing parameters and calculations is achieved without affecting the accuracy of model recognition. The specific process is shown in Figure 8:

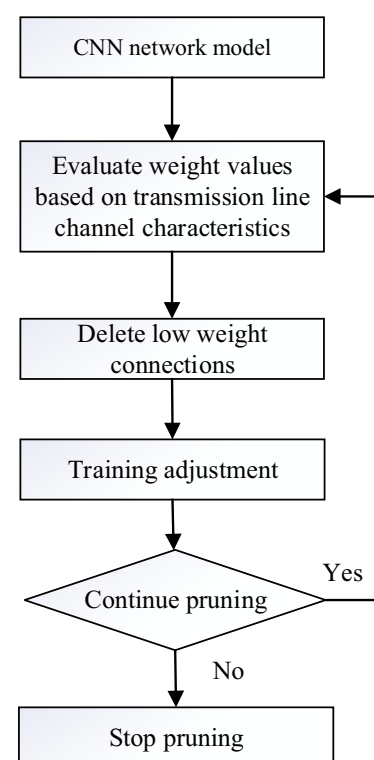

Figure 7. Pruning process

The model quantization adopts the tensorRT scheme. The float 32bit convolution operation is converted to the int8 convolution operation, and then performs linear quantization, and the mapping relationship satisfies formula (2):

$$
\begin{aligned}
& \text { FB32 Tensor }(T)=\text { scale_factor }(s f) \times \\
& \text { 8bitTensor }(t)+\text { FB32_bias }(b)
\end{aligned}
$$

And sf is the scale factor of each tensor in each layer, and $\mathrm{T}$ is the calculation threshold. Map $\pm|\mathrm{T}|$ to \pm 127 , where $|\mathrm{T}|<|\max |$. Values beyond the threshold $\pm|\mathrm{T}|$ are directly mapped to \pm 127 , that is, the direct mapping beyond -127 is -127 , and the direct mapping beyond 127 is 127 . Choose a suitable threshold and discard only the larger values of the scattered distribution to ensure the lowest loss of accuracy [5-7].

\section{Design verification}

There are more than 11,000 typical pictures of the external force destruction scenes, covering five categories of tower cranes, cranes, construction machinery, wildfire smoke, and line foreign objects. It is divided into more than 9,000 training sets and more than 2,000 test sets. Based on the lightweight algorithm model of the training set, the recognition accuracy in the test set is shown in Table 1:

Table 1. Identification index

\begin{tabular}{|c|c|c|c|c|c|}
\hline Index & Tower crane & Crane & $\begin{array}{c}\text { Construction } \\
\text { machinery }\end{array}$ & Wildfire smoke & Line foreign objects \\
\hline Training set & 2672 & 1905 & 2847 & 893 & 936 \\
\hline Test set & 1120 & 753 & 1241 & 297 & 304 \\
\hline Accuracy & $92.6 \%$ & $87.1 \%$ & $92.8 \%$ & $69.8 \%$ & $63.2 \%$ \\
\hline False alarm rate & $4.8 \%$ & $5.4 \%$ & $3.7 \%$ & $11.4 \%$ & $15.8 \%$ \\
\hline Omission rate & $1.7 \%$ & $3.2 \%$ & $2.1 \%$ & $7.5 \%$ & $8.7 \%$ \\
\hline
\end{tabular}


Indicator description:

(1) Accuracy = correct number of target recognition / standard target number of test set.

(2) False alarm rate $=$ number of identified images without hidden dangers / total number of images detected.

(3) Omission rate $=$ number of hidden but unidentified pictures/total number of pictures with hidden dangers.

\section{Conclusion}

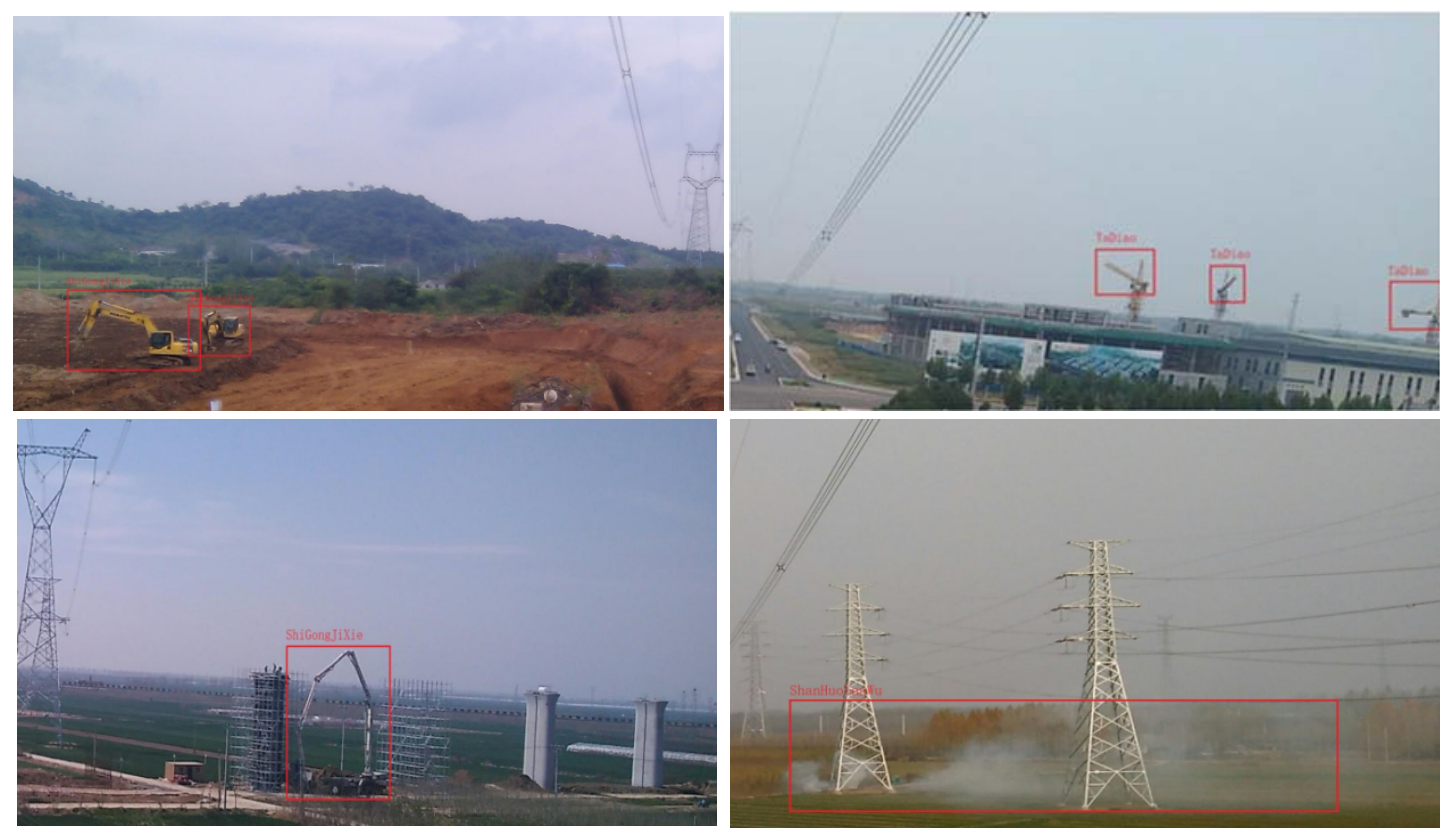

Figure 8. Application recognition effect

\section{Acknowledgments}

Acknowledge assistance and encouragement from colleagues. Thanks for assistance from the technical staff of Province-level Power Grid in the pilot application.

\section{References}

1. Gong, J.Z. (2012) Cause analysis and prevention countermeasures of external breakage of transmission lines. Hunan Electric Power, 32: 3638 .

2. Lu,J.Z., Zhou,T.J. Wu,C.P., Li,B., Tan,Y.J., Zhu,Y. (2016) Fault Statistics and Analysis of $220 \mathrm{kV}$ and Above Power Transmission Line in Province-level Power Grid. High Voltage Engineering, 42: 200-207.

3. Xu, Z.L., Zeng, Y.H., Guo, S., Saho, X.J., Mai, J.J., Hu, Z.L. (2020) Application of Intelligent Monitoring System for Transmission Lines
More than 10 equipment are installed on the high-voltage time identification of the external force destruction. When the risk of the external force destruction is found, the alarm data is pushed to the server in real time. The server notifies the operation and maintenance staff and starts early warning measures. The system operates stably in complex weather conditions and can meet the needs of field applications. The recognition effect is shown in Figure 8. transmission line of Province-level Power Grid, for real-

Based on Image Recognition Technology. Computer Systems \& Applications, 29: 67-72.

4. Hu,F.Y., Li,L.Y., Shang,X.R., Shen,J.Y., Dai,Y.L. (2020) Overview of target detection algorithms based on convolutional neural networks. Journal of Suzhou University of Science and Technology (Natural Science, 37: 1-10.

5. Bi,P.C., Luo,J.X., Chen,W.W. (2019) Research on Lightweight Convolutional Neural Network Technology. Computer Engineering and Applications, 55: 25-35.

6. Chen, C.X. (2019) Research of Lightweight Convolutional Neural Network. South China University of Technology.

7. Luo,M.Y., Liu,Y.F. (2019) Clothing Classification Method Based on Lightweight Convolutional Neural Network. Computer Systems \& Applications, 28:223-228. 